\title{
Argumentative Procedures in e-Commerce Environments
}

\author{
Paulo Novais, José Neves, Luís Brito and José Machado \\ Universidade do Minho \\ Departamento de Informática \\ Campus de Gualtar \\ 4710-057 Braga, PORTUGAL
}

\begin{abstract}
The use of agent's technology in electronic commerce environments leads to the necessity to introduce some sort of formal attitude in the processes of software development and analysis. Logic programming and specially extended logic programming provides a powerful tool for achieving such goal; i.e., besides being mathematically correct it makes the prototyping phase easier. However, although no simple methodology had yet been stated to address the complexity present in this approach, it not only addresses problems of architecture development and analysis, but also looks at problems of knowledge representation and reasoning, and machine learning. Such a framework will follow the Experience-Based Mediator agent paradigm particularly suited to take into account the argumentation schemes that are inherent to any electronic commerce deal.
\end{abstract}

Key words: electronic commerce, intelligents agents, extended logic programming, preargument reasoning and argument-based negotiation.

\section{INTRODUCTION}

The amount of ambiguity present in real-life deals has to be brought to a minimum, specially in the process that opposes, during negotiation, intelligent entities (i.e., the agents), here understood as automatic theorem provers. Logic, and especially Extend Logic Programming (ELP) [Baral/ Gelfond, 1994] poses itself as a powerful tool to achieve both the desired formality without compromising comprehension/readability, and the ability to easily build an executable prototype. However, in a dynamic environment

\footnotetext{
The original version of this chapter was revised: The copyright line was incorrect. This has been corrected. The Erratum to this chapter is available at DOI: 10.1007/978-0-387-35617-4_48 
such as the one found in Electronic Commerce (EC), the simple use of logical formula is not enough. It is paramount to look at formal systems that encapsulate non-monotonous behaviour. An agent believes that something is true at a given point in time but it may conclude differently at a later time. In ELP programs, queries may not be answered with a simple true or false statement. To represent incomplete knowledge about the world, the possibility for the unknown answer there exists. To enable this to happen, ELP programs endorse two kinds of negation: $\neg$ (called classical, strong or explicit negation) and not (called negation-by-failure). For programs in logic programming, negative information is provided by the closed-world assumption (i.e., everything that can not be proven to be true is false); however, in extended logic programs, that is not quite so. In ELP a query may fail due to the fact that information is not available to support it, or it may fail due to the fact that negation succeeds [Baral/Gelfond,1994], [Neves/Machado/Analide/Novais/Abelha, 1997]. The use of logic, in order to state the reasoning mechanisms behind the negotiation process, complements previous work in the development of a formal framework for EC-directed agents [Brito/Novais/Neves, 2001]. The reasoning procedures that set the behaviour of each agent follow a quantification process of attitudes (i.e., a mental view or disposition) such as agreement and gratitude [Brito/Novais/Neves, 2001], and gives way to a formal definition of the argumentative process [Brito/Novais/Neves, 2001]. The strategy for developing a consistent and sound approach to the use of agents in EC is based on a constructive view of the problem-solving scenario [Novais/Brito/Neves, 2001], in terms of:

- Architecture development: to determine and analyse each of the features an agent should have to deal with a particular area of interest; to design the flow of information across the different agent building blocks [Novais/Brito/Neves, 2000];

- Process quantification: to quantify each metric and sub-process with which the agents have to deal with [Faratin/Sierra/Jennings, 1997], [Brito/Novais/Neves, 2001];

- Reasoning procedures: each agent is in need of a set of axioms that may serve as the main guidelines for the negotiation processes [Norman/Sierra/Jennings, 1998]. i.e., set of factors are to be taken into account/evaluated, before any kind of opposition among agents in a Virtual Market (VM) may take place [Brito/Novais/Neves, 2001]; and

- Process formalization: argumentation is in need of a formal treatment in order to be consistent and for the agents to act/react in a reasonable way [Brito/Novais/Neves, 2001]. On the other hand setting an argument in a business environment has many similarities to what happens in the legal arena [Prakken, 1993], [Sartor, 1994]. 
Some dispositions such as temporality and priorities were formally addressed in [Brito/Novais/Neves, 2001], which are taken to be central for any agent that may be engaged in a deal. Temporality introduces a time tag (temporal validity) on the base clauses in the $\mathrm{KB}$, something that is to be considered when one is looking at the non-destructive (non-monotonic) principles of knowledge processing. Priorities are useful when reasoning over the set of base clauses in order to justify/explain some course of action. Although the use of agent-based systems in EC is not new, especially at the negotiation level, a problem's solution has been hampered in the past by the lack of a formal basis, in terms of software development and analysis.

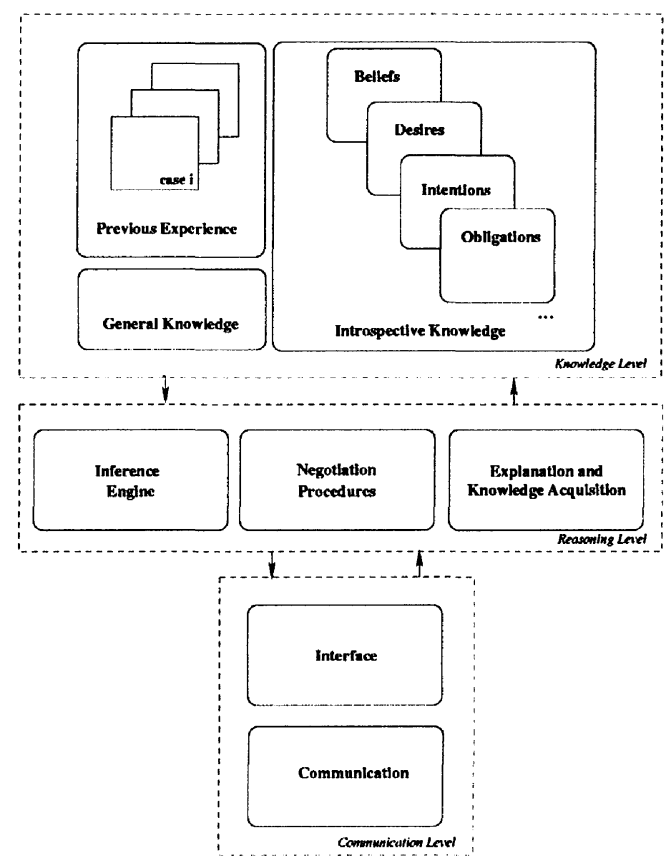

Figure: The Layout of an EBM's Agent

\section{ARCHITECTURE DEVELOPMENT}

The Experience-Based Mediator Agent (EBM) [Novais/Brito/Neves, $2000]$ is used as the provider of a logic-based framework for pre-argument reasoning and argument generation. However, it must be stated that agents oriented to price manipulation based on econometric approaches represent interesting alternative solutions to the same problem (although with limited reasoning capabilities) [Kephart/Hanson/Greenwald, 2000]. The EBM agent is a general module-oriented architecture aiming at the development of intelligent agents for EC (Figure). Taking previous experiences as a starting 
point, the agent's knowledge base is complemented by general and introspective knowledge, the former comprises information about the system itself and/or the prices and rules followed by counterpart agents, the last embraces anthropopatic passions such as beliefs, desires, intentions and obligations. An agent must be able to reason about general or even incomplete information, on the one hand, and it must also be able to explain its own behaviour or acquire new knowledge, on the other hand. But, in the present context, these procedures are not enough. The ability to deal with the market's specificities is paramount (e.g., the ability to form prices, to evaluate a good or service or to cartelise [Novais/Brito/Neves, 2000], [Brito/Novais/Neves, 2001]).

\section{THE PRE-ARGUMENTATIVE REASONING PROCESS}

The set of some of the most important features that intervene in the negotiation process will now be object of study, in terms of gratitude, delegation and agreement (taking into account temporality and priority rules) [Brito/Novais/Neves, 2001]. The general process of negotiation must be clearly distinguished from the argumentative one [Brito/Novais/Neves, 2001]. The process of argumentation is tightly coupled with the process of logically founded attack on the arguments put forward by a counterpart. It works with price-formation issues and deal finalization; negotiation is a wider concept that is coupled with specific forms of reasoning, dealing with the high-order, pre-arguing relationships, that may be established among agents.

The Knowledge Base (KB) of an agent is made of a series of facts and rules. Facts provide the basic information, set the agent's object knowledge level, and feed the mechanisms at the meta level in order to make an agent to behave properly. In this work it is assumed that it has the ability to deal with every product or service, under any scenario. However, any agent has its behaviour conditioned by the right to deal. Predicates like capability-to-deal: Product or service, Conditions, Counterpart $\rightarrow$ \{true, false\}, and right-todeal: Product, Conditions, Counterpart $\rightarrow$ \{true, false\} (where Product, Conditions and Counterpart stand, respectively, for the product or service to be traded, the conditions associated to that operation and, the counter-part agent involved in the deal) lead one to differentiate between the capacity to deal with the ability to deal (i.e., an agent is always able to deal a product or service, but it may be restricted to do so due to some kind of invariant or restriction). 
Definition 1. A logical Theory for Negotiation Agents is defined as the quadruplet $T N A=<R, C, B P, \pi>$ where $R, C, B P$ and $\pi$ stand, respectively, for the set of predicates on the right-to-deal, the set of invariants $(A:+$ restriction::P), the set of behavioural predicates (including the theorem prover) and a non-circular order relation that states that if $P \pi Q$, then $P$ occurs prior to $Q$.

Factual clauses are represented by productions of the form $\mathrm{ag}_{\mathrm{x}}: \mathrm{P}$, and rule clauses by $\mathrm{ag}_{\mathrm{x}}: \mathrm{P} \leftarrow \mathrm{Q}$. Invariants are represented at the agent's $\mathrm{KB}$ through clauses of the form A:+restriction::P, where $\mathrm{A}$, +restriction and $\mathrm{P}$ denote, respectively, an agent's identification, the invariant's type and the invariant itself. A set of definitions that incrementally state the formal basis for the pre-argumentative reasoning process will now be described.

Definition 2. A LP Theorem Prover for Incomplete and Temporal Information with built-in Priorities. Taking factual clauses with temporal validity, $a$ body of knowledge classification (represented by $\left.B K:: P::\left[i_{1}, i_{2}, \ldots, i_{n}\right]\right)$ and rule clauses (represented by $P \leftarrow Q$ ) as the components of the $K B$ present into each agent Knowledge Base, the predicate demo $o_{L P T P}: T, C T, V \rightarrow$ \{true,false\}, where $T, C T, V$ and \{true, false\} stand, respectively, for a logical theorem, the current time, and the theorem valuation, denotes the theorem prover for incomplete and temporal information over $K B$, in terms of the following set of rules:

$$
\begin{aligned}
& \operatorname{demo}_{L P I T P}(P, C T, \text { true }) \leftarrow \quad \operatorname{priority}\left(B K_{1}, B K_{2}\right) \text {, } \\
& \text { test } t_{\text {priority }}\left(B K_{1}, B K_{2}, P, T\right) \text {, } \\
& \text { in }_{\text {time }}(C T, T) \text {. } \\
& \operatorname{demo}_{L P I T P}(P, C T, \text { false }) \leftarrow \quad \text { priority }\left(B K_{1}, B K_{2}\right), \\
& \text { test }_{\text {priority }}\left(B K_{1}, B K_{2}, P, T\right) \text {, } \\
& \neg \text { in }_{\text {time }}(C T, T) \text {. } \\
& \operatorname{demo}_{L P I T P}(P, C T, \text { false }) \leftarrow \quad \text { priority }\left(B K_{1}, B K_{2}\right) \text {, } \\
& \neg \text { test }_{\text {priority }}\left(B K_{1}, B K_{2}, P, T\right) \text {, } \\
& \text { in }_{\text {time }}(C T, T) \text {. } \\
& \operatorname{demo}_{L P I T P}(P,-, \text { unknown }) \leftarrow \quad \operatorname{priority}\left(B K_{1}, B K_{2}\right) \text {, } \\
& \text { not test } t_{\text {priority }}\left(B K_{1}, B K_{2}, P,-\right) \text {, } \\
& \text { not } \neg \text { test }_{\text {priority }}\left(B K_{1}, B K_{2}, P,-\right) \text {. } \\
& \text { test }_{\text {priority }}\left(B K_{1},-, P, T\right) \leftarrow \quad\left(B K_{1}: \because P: \because T\right) \text {. } \\
& \text { test } t_{\text {priority }}\left(-, B K_{2}, P, T\right) \leftarrow \quad\left(B K_{2}: \because P:: T\right) \text {. } \\
& \rightarrow \text { test }_{\text {priority }}\left(B K_{1},-, P, T\right) \leftarrow \quad \neg\left(B K_{1}: \because P: \because T\right) \text {. } \\
& \rightarrow \text { test }_{\text {priority }}\left(-, B K_{2}, P, T\right) \leftarrow \quad \neg\left(B K_{2}:: P:: T\right) \text {. }
\end{aligned}
$$

where predicates in time: $: C T, L T \rightarrow\left\{\right.$ true, false\}, test $t_{\text {priority: }}: B K_{a}, B K_{b}, P$, $T \rightarrow\{$ true, false $\}$ and $\rightarrow$ test priority: $B K_{a}, B K_{b}, P, T \rightarrow\{$ true, false $\}$ stand for themselves. 


\subsection{Gratitude}

A characterization of the marginal gratitude concept is depicted in [Brito/Novais/Neves, 2001], in terms of non-negotiable gratitude and negotiable gratitude. Analysing this two situations it can be seen that the former (non-negotiable gratitude) occurs with gratitude value Value (in predicate gratitude()), taking into account the Negotiation Information (NI), the counterpart agent $(Y)$ and the present time $(C T)$, a specific offer (e.g., a gift) is delivered from the counterpart agent. The last situation (negotiable gratitude) occurs whenever it is possible to deal with a specific agreement in turn, states in its own $\mathrm{KB}$ that the former is able to drop a specific negotiation (probably a competitive negotiation) in exchange for some kind of compensation (reflected in terms of gratitude). In formal terms, this may be stated as it is depicted below:

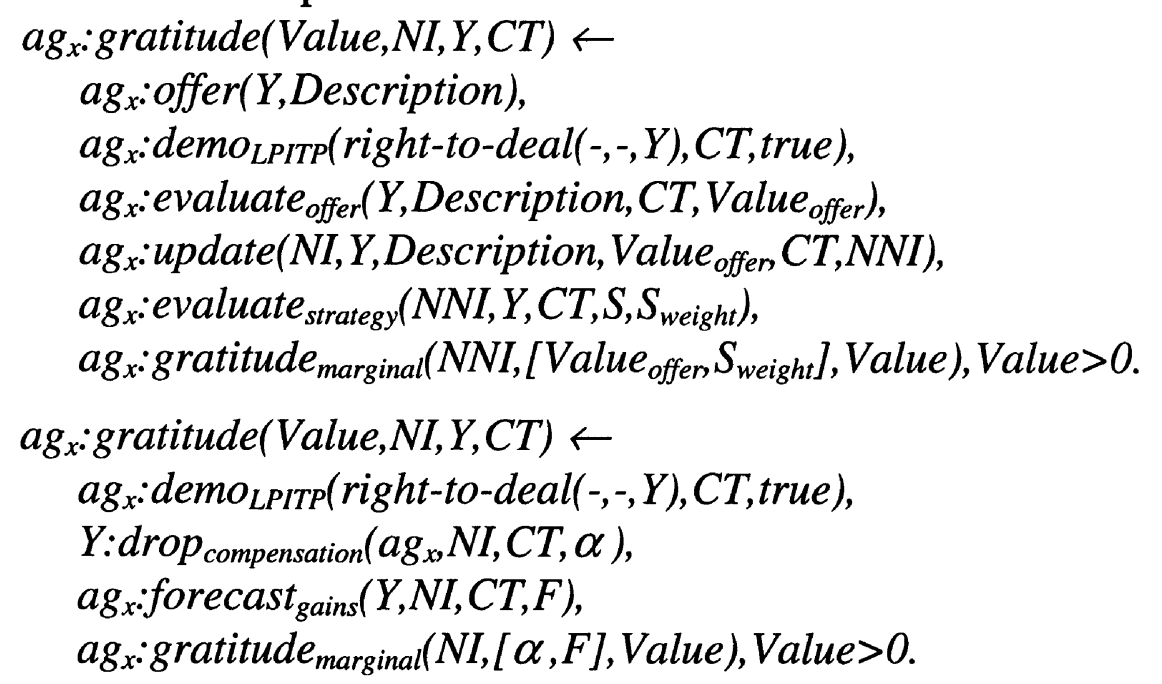

\subsection{Delegation}

Delegation can be seen as the task of chosen or electing somebody or someone to act for or represent another or others on a negotiation. Negotiation tasks may only be delivered to a third party if there is sufficient knowledge relating to the right to deal. Delegation acts as a way to undertake indirect negotiations; i.e., it uses a proxy agent taking advantage of its particular characteristics, such as gratitude debts and agreements established among the proxy and the other agents [Brito/Novais/Neves, 2001]. A formal characterization of the problem is given by considering that delegation involving a product or service $\mathrm{P}$, conditions $\mathrm{C}$ and counterpart $\mathrm{CP}$ to agent $\mathrm{Y}$ at time $\mathrm{CT}$, is possible if the representative's agent is able to deal the product or service with the counterpart; the delegable one is able to deal with the proxy agent; and the proxy agent is able to deal with its counterpart by itself. In formal terms, one may have: 


$$
\begin{aligned}
& a g_{x}: \text { delegate }(P, C, C P, Y, C T) \leftarrow \\
& a g_{x}: \text { demo }_{L P I T P}(\text { right-to-deal }(P, C, C P), C T, \text { true }), \\
& a g_{x}: \text { demo }_{L P I T P}(\text { right-to-deal }(P,-, Y), C T, \text { true }), \\
& Y: \text { valid }_{\text {assimilation }}(Y: \text { right-to-deal }(P, C, C P), C T) .
\end{aligned}
$$

\subsection{Agreement}

Like gratitude, agreement can be understood as a subjective attitude that introduces non-linearities in the negotiation process [Brito/Novais/Neves, 2001]. The simplest case of agreement is the one that relies in the absolute majority of votes to beat the combined opposition or the runner-up. This democratic approach relies on the existence of fully veridical agents; i.e., agents that convey their opinion in a consistent manner to their peers [Novais/Brito/Neves, 2000]. An agreement can only be reached among entities that are able to deal with each other; i.e., if an agent is unable to assert the right to deal with other agent(s), it can never establish some sort of commitment (agreement). An agreement is reached on a specific Subject (S), among a set of Entities (E) with a set of Opinions (O) at a specific time (CT). By definition, an agent is in agreement with itself on every subject. In formal terms, one may have:

$$
\begin{aligned}
& \text { ag: agreement }\left(-,\left[a g_{x}\right],-,-\right) \text {. } \\
& \text { ag: agreement }(S, E, O, C T) \leftarrow \\
& a g_{x}: \text { can-deal-with }(E, C T) \text {, } \\
& a g_{x}: \text { gather }_{\text {opinions }}(S, E, C T, L O) \text {, } \\
& a g_{x}: \text { summarize }(S, O, L O, C T) \text {. } \\
& \text { ag } x \text { :can-deal-with }([A], C T) \leftarrow \\
& \text { ag: } \text { demo }_{\text {LPITP }}(\text { right-do-deal(-,-,A),CT,true). } \\
& \text { ag: can-deal-with }([A \mid T], C T) \leftarrow \\
& a g_{x}: \text { demo }_{\text {LPITP }}(\text { right-do-deal(-,-,A),CT, true), } \\
& a g_{x}: \text { can-deal-with }(T, C T) \text {. }
\end{aligned}
$$

\section{LOGIC-BASED ARGUMENTATION}

When a set of agents (e.g., EBM agents) meets under a Virtual Marketplace (VM), some kind of interaction may take place, namely by a process of offers and counter-offers, to support the modelling, analysis and enactment of the business process. The soundness of the process arises from the set of facts taken into consideration to produce an offer or counter-offer; i.e., the facts taken from an ordered logic theory, lead to a logical conclusion, organizing themselves into an argument. The importance of an argument has much to do with the time at which it arises; i.e., an argument 
may be deemed as a looser or a winner when facing a counter-argument, taking into account its sequence of evaluation. The exchange of offers and counter-offers must stop when some conditions are satisfied. These conditions may or may not lead to the definition of a winning set of arguments, which is the case in systems where the main concern goes to take full advantage of the arguments' valuation.

\subsection{Global versus Proper Knowledge}

Each term that is part of an argument may come from one of two main sources: global or proper knowledge. Global knowledge is shared by the intervening entities and is, therefore, independent of a particular experience. Proper knowledge derives from sources that are not common to every agent, giving way to the possibility of contradictory conclusions upon confrontation. Contrary to the practices found in the logical approaches to the application of the The Law [Sartor, 1994], the KB embedded in each agent may have a quite different genesis. The use of global or proper knowledge is a conditioning of the system's capacity to determine the winner/loser of a confrontation. As expected, proper knowledge is not the best starting-point for a premise denial/attack (e.g., a claim such as $m y$ experience tells me I sold item X for $Y$ euro is difficult to be stated as false by a counterpart, once he/she can not say which is the grasp of life of the opponent).

\subsection{The Structure of an Agent's Knowledge Base}

Prior to the characterization of the argument's structure in terms of ELP productions, the agent's knowledge base has to be addressed. It will be built around a set of logical clauses subject to proof, then allowing for action justification and argument construction.

Definition 3. The knowledge available in each agent's $K B$ is made of logic clauses of the form $r_{k}: P_{i+j+1} \leftarrow P_{1 \wedge} P_{2 \wedge} \ldots \wedge P_{i-1 \wedge}$ not $P_{i \wedge} \ldots \wedge P_{i+j}$, where $I, j, k \in N_{0}, P_{l}, \ldots, P_{i+j}$ are literals; i.e., formula of the form $p$ or $\neg p$, where $p$ is atom, and where $r_{k}$, not, $P_{i+j+1}$, and $P_{1 \wedge} P_{2 \wedge} \ldots \wedge P_{i-1}$ not $P_{i \wedge} \ldots \wedge$ $P_{i+j}$ stand, respectively, for the clause's identifier, the negation-as-failure operator, the rule's consequent, and the rule's antecedent. If $i=j=0$ the clause is called a fact and is represented as $r_{k}: P_{l}$.

An ELP program ( $\Pi_{\mathrm{ELP}}$ ) is seen as a set of clauses as the ones given by Definition 3. Therefore, the KB of an agent is taken from an ordered theory $\mathrm{OT}=(\mathrm{T},<,(\mathrm{S}, \pi))$, where and stand, respectively, for an agent's knowledge base in clausal form, a non-circular ordering relation over such clauses, a set of priority rules, and a non-circular ordering relation over such rules. An 
argument (i.e., a proof, or series of reasons in support or refutation of a proposition) or arguments have their genesis on mental-states seen as a consequence of the proof processes that go on unceasingly at the agent's own knowledge about its states of awareness, consciousness or erudition. On the other hand the mental states that have been referred to above are by themselves a product of reasoning processes over incomplete or unknown information; an argument may not only be evaluated in terms of true or false, but it may be quantified over the interval [ $0, \infty[$ (e.g., agent is able to deal product with agent using the set of conditions $\mathrm{C}_{1}$; however it is not known if it can do the same thing with a set $\mathrm{C}_{2}$-leading to further confrontation).

Definition 4. Taking ordered theory OT, an argument is a finite, nonempty sequence of terms $\left\langle r_{1}, \ldots, r_{n}\right\rangle$ such that, for each sequence rule $r_{j}$ with $P$ as a part of the antecedent, there is a term $r_{i}(i<j)$ on which the consequent is $P$.

It must be taken into consideration that in Definition 4, in the non-empty sequence of terms $\left\langle\mathrm{r}_{1}, \ldots, \mathrm{r}_{\mathrm{i}}, \ldots, \mathrm{r}_{\mathrm{n}}\right\rangle, \mathrm{r}_{\mathrm{i}}$ stands for a call to a proof's predicate, given in the form $\operatorname{demo}\left(r_{i}, v_{i}\right)$, that establishes the truth, validity or quality of $r_{i}$ in terms of $v_{i}$.

Definition 5. In a argument (i.e., a proof, or series of reasons in support or refutation of a proposition), the last reason (i.e., the faculty of rational argument, deduction, judgment) in support or refutation of a proposition relates to the consequent of the rule used in such a situation. Such a consequent brings the parts together in terms of the argument's judgment.

According to Definition 5, it is possible to build on arguments that are in opposition; i.e., where there are rules that attack (or deny) previous stated propositions. Taking into account the two forms of argument attack (conclusion denial and premise denial), a conflict among two opposing agents (e.g., buyer/seller) can be formally specified.

Definition 6. Conflict/attack over negotiation arguments. Let $A_{1}=\left\langle r_{1,1}, \ldots, r_{1, n}\right\rangle$ be the argument of agent 1 and $A_{2}=\left\langle r_{2,1}, \ldots, r_{2, m}\right\rangle$ be the argument of agent 2 . Then,

(1) if $r_{1, i} \in A_{1}$ or $r_{2, j} \in A_{2}$ are local, the arguments are said to be in "probable conflict";

(2) $A_{1}$ attacks $A_{2}$ iff $A_{1}$ executes a conclusion denial attack or a premise denial attack over $A_{2}$;

(3) $A_{1}$ executes a conclusion denial attack over $A_{2}$ iff there is no local knowledge involved and conc $\left(A_{1}\right)$ is contrary to conc $\left(A_{2}\right)$; and

(4) $A_{1}$ executes a premise denial attack over $A_{2}$ iff there is no local knowledge involved and conc $\left(A_{1}\right)$ is contrary to some $r_{2, j} \in A_{2}$.

Having in mind the use of rational agents (i.e., those that do not undermine their own actions and are able to formulate coherent arguments), 
a definition of coherency may be formulated and, on the other hand, posterior definitions for victory/defeat (which agent, if any, wins or looses an argument-based negotiation) must take into account this formulation.

Definition 7. An argument $A_{1}=\left\langle r_{1}, \ldots, r_{n}\right\}$, where $r_{k}(k=1, \ldots, n)$ are arguments, is said of being built around coherent's arguments iff $\neg \exists\left(r_{i}, r_{j}\right)$ with $r_{i}, r_{j} \in A$ and $i \neq j$, such that $r_{i}$ attacks $r_{j}$.

where iff stands for if and only if, $\neg$ denotes strong negation and $\epsilon$ denotes the set's inclusion operator.

At this stage a new concept is need, that of round. Indeed, all the logical definitions presented take into account a mutual exclusion principle over the set of active agents; i.e., at each turn, an agent expresses its judgment to a counterpart. This order, defined by the round structure, implicitly states the direction of the attacks and the victory/defeat directions (if it is $A_{1}$ that defeats $A_{2}$, or $A_{2}$ that defeats $A_{1}$ ). Therefore, taking into account the definition of conflict/attack and the concept of round it is possible to caracterize define the victory/defeat pair.

Definition 8. Victory/defeat of negotiation arguments. Let $A_{1}=\left\langle r_{1,1}, \ldots, r_{1, n}\right\rangle$ be the argument of agent 1 and $A_{2}=\left\langle r_{2,1}, \ldots, r_{2, m}\right\rangle$ be the argument of agent 2 and $A_{2}$ is presented at a later "round" than $A_{1}$. Then, $A_{1}$ is defeated by $A_{2}$ (or $A_{2}$ is victorious over $\left.A_{1}\right)$ iff

(1) $A_{2}$ is coherent and $A_{1}$ is incoherent;

(2) $A_{2}$ is coherent, executes a conclusion denial attack over $A_{I}$ (coherent) and the conclusion of $A_{2}$ is prioritary (taking into account the Theory for Negotiation Agent with Priorities TNAP) over $A_{1}$; and

(3) $A_{2}$ is coherent, executes a premise denial attack over $A_{1}$ (coherent) and the conclusion rule of $A_{2}$ is prioritary (taking into account the TNAP theory) over $A_{1}$.

\subsection{Examples}

Some examples may now be presented to illustrate such a framework. Let agents $\mathrm{E}$ and $\mathrm{F}$ be engaged in the process of buying/selling product $\mathrm{p} 1$ in an environment with priority rules embedded in the KBs. Agents $\mathrm{E}$ and $\mathrm{F}$ share general knowledge, market knowledge and the set of priority rules.

Agent E:

$P E: r_{5}: \operatorname{price}(p 1,143)$.

$\%$ (experience) price for $p 1$ is 143

$M K: r_{7}:$ price $(p 1,147)$.

$\%$ (market) price for pl is 147

$G K: r_{1}: \operatorname{price}(p 1,150)$.

$\%$ (global) price for pl is 150

$P R I O: r_{4}$ : priority $(P E, G K)$.

$\%$ (priority) PE overpowers $G K$

$P R I O: r_{6}: \operatorname{priority}(M K, P E)$.

$\%$ (priority) PE overpowers $G K$

Agent F: 


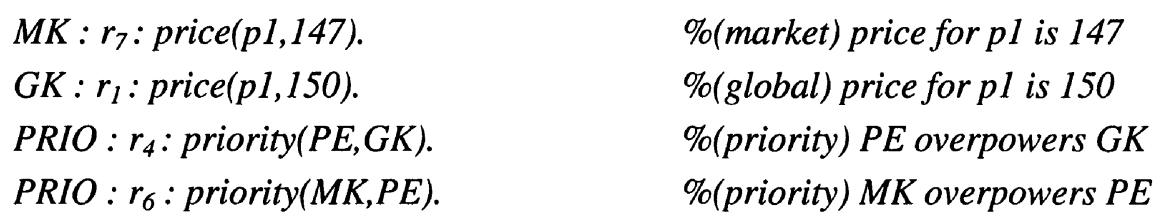

The argument given by agent $\mathrm{E}$ might be $\mathrm{A}_{\mathrm{E}}=\left\langle\mathrm{r}_{4}, \mathrm{r}_{5}\right\rangle$; however, agent $\mathrm{F}$ might argue with $A_{F}=\left\langle r_{6}, r_{7}\right\rangle$, in terms of a conclusion denial attack, taking into account the priority rules shared by the community. Agent $\mathrm{F}$ is named the winner due to the fact it uses an higher priority rule.

An example of the use of incomplete information in the generation of argument is now considered, taking into account the statement of interest in the purchase of $\mathrm{p} 1$ (which is an imported good) from agent J. Agent I intends to state that its interest in that purchase is unknown (in order, perhaps, to ignite a price reduction by the counterpart -- a simple information exchange situation). The KB of agent I may therefore be depicted as follows:

Agent I:

$\begin{array}{ll}G K: r_{1}: \text { sells }(\text { agentJ,importedgood }) . & \%(\text { global }) \text { agent } J \text { sells an imported good } \\ G K: r_{2}: \text { imported }(p 1) . & \%(\text { global }) \text { pl is imported } \\ L K: r_{3}: \text { interested }(S, P) \leftarrow & \%(\text { local }) \text { interest in product } P \\ -:-: \operatorname{sell}(S, P) . & \% \text { sold by } S \\ L K: r_{4}:- \text { interested }(S, P) \leftarrow & \%(\text { local }) \text { not interested in product } P \\ \text { not }-:-: \text { interested }(S, P), & \% \text { sold by } S \text { if interest or exception } \\ \text { not }-:-: \text { exception }(S, P) . & \% \text { fail to be proved } \\ L K: r_{5}: \operatorname{exception}(S, P) \leftarrow & \%(\text { local }) \text { exception if product } P \\ -:-: \operatorname{sell}(S, \text { importedgood }), & \% \text { is imported and } S \text { sells some } \\ -:-: \text { imported }(P) . & \% \text { imported good }\end{array}$

The argument generated by $I$, taking the previous $K B$ into account, might be $A_{1}=\left\langle r_{1}, r_{2}, r_{3}, r_{5}, r_{4}\right.$, demo(interested(agentJ,pl), unknown) $\rangle$. This argument provides new information to the counterpart agent, upon which some action may be taken.

\section{ARGUMENT GENERATION}

The use of logic in the specification of argument-based negotiation aims not only at the definition of the best dealing strategies but also to mimic the inference mechanisms that take place in the mind of the user, in order to enable a true feasible implementation of agent-based argumentation for EC. Automatic argument's generation relies on three types of proceedings:

- proto-autonomy: at each round the user redefines the global course of action (e.g., attack/denial) and only then the incumbent agent indicates which arguments (first in time, order or rank) are to be launched; 
- semi-autonomy: to the system's agents must be granted access to historical arguments based databases, in order to allow the incumbent's agents to use different data or knowledge handling techniques to extrapolate the intended course of action when a new argument is on the table; and

- full-autonomy: using Machine Learning tools the incumbents agents may progressively learn and adapt their mental states to a particular sort of arguments which are exchanged.

The process of argument generation may be seen as a logic-guided mechanism that acts in order to support some utterance (e.g., it is possible for an human being to act in support of an argument, obtained on the basis of the formalism referred to above, when a KB of local or global knowledge is made available (to him/her)) [Novais/Brito/Analide/Neves, 2002].

\section{MAIN CONTRIBUTIONS}

The main contributions present of work are listed below: (i) the definition of a common ground to situate the agent's reasoning mechanisms in EC environments; (ii) the use of formal tools (logic) to describe the rational behaviour of agents involved in EC; (iii) the description of a reasoning mechanism necessary for a consistent and sound development of agents for EC; (iv) the use of incomplete information and previous experiences in the reasoning process; (v) the bridging of legal argumentation and argumentbased negotiation; (vi) setting the distinctive and most interesting characteristics of logic-based argumentation; and (vii) the establishment of sound syntactic and semantic tools for logic-based argumentation.

\section{RELATED WORK}

The work of Prakken [Prakken, 1993] and Sartor [Sartor, 1994] (among others), served as the basis for the development of an argument-based formal framing for EC-based agents; indeed, the relation established between legal argument and legal language, especially when applied to problems of delegation or liability make the bridge for the world of automated commerce. The establishment of an agent architecture that suits the logical characteristics imposed by the present model were studied in the work of Novais et al [Novais/Brito/Neves, 2000]. The EBM agent is suitable either as the basis for agents that go to The Law (i.e., that resort to legal proceedings on argument building), or for the development of a formal foundation for argumentation. Argumentation has been approached in [Jennings/Parsons/Noriega, 1998] as a way to navigate through each agents 
accepted area of influence in an informal manner, leading to information exchange among agents, then increasing the probability of possible settlements. The present framework (agent architecture/argument building) establishes a formal and consistent bridge between argumentation as it is seen in The Law and argument-based negotiation [Brito/Novais/Neves, 2001], [ Novais/Brito/Analide/Neves, 2002]. A working prototype of such a system can be found in [Brito/Novais/Neves, 2001], [Novais/Brito/Neves, 2001].

\section{CONCLUSIONS}

In EC the introduction of agent-based technology makes possible the use of a set of high-level reasoning tools, leading to architectures that base themselves on the assumption that in a real-world environment entities act as mediators. Under this umbrella, simple inference and knowledge acquisition mechanisms are not sufficient to provide a sustainable and credible system. Traditional computational models need to be complemented with procedures native to the business. Indeed, the issues posed by EC need to be addressed in a formal fashion. EC is an area that poses particular problems to the use of agent-based software. Although applications in this area are particularly suited to be solved by agent's based systems, no formal development process has yet been devised for such field of expertise. In this work, the problem solving process starts with the definition of an agent architecture, then the processes among agent's are set and quantified, and the reasoning mechanisms are recognized. The EBM's agent architecture was developed, considering functionalities that are specially suited to deal with situations brought in by past-experiences, a feeling of thankfulness or appreciation (i.e., gratitude) or a settlement (i.e., agreement). The processes involved in $\mathrm{EC}$, which are difficult to incorporate into traditional systems, revolve around subjective business parameters. Indeed, parameters such as gratitude and agreement among parties are non-linearities, which need to be considered in order to develop a feasible EC system. This information is to be taken into account when drawing up a strategic plan of action. However, once subjective parameters have been quantified, some reasoning must take place before any argument is exchanged with potential counterparts. This stage, which has been called pre-negotiation reasoning deals with the existence of incomplete information and delineates logical conclusions upon an agent's $\mathrm{KB}$ (e.g., is agent $\mathrm{A}$ able to deal product $\mathrm{P}$ with agent $\mathrm{B}$ at time $\mathrm{T}$ ). Exchanging justified information provides an agent's counterpart with enough knowledge to try a common understanding with its counterpart in 
time. Specifying the way an agent can attack an argument culminates the most important phase in the development of EC-directed agent software.

Acknowledgments: The work of José Neves was carried out under the project POSI/EEI/13096/2000.

\section{REFERENCES}

Baral, C., Gelfond, M., Logic Programming and Knowledge Representation, Research Report, Computer Science Department, University of Texas at El Paso, USA (1994).

Brito L., Novais P., Neves J., Temporality, Priorities and Delegation in an E-Commerce Environment. Proceedings of the 14th Bled Electronic Commerce Conference, Slovenia (2001).

Brito L., Novais P., Neves J., Mediation, Agreement and Gratitude in Strategic Planning for Virtual Organizations. Enterprise Information Systems II, Ed. Sharp B., Filipe J., and Cordeiro J., Kluwer Academic Publishers (2001).

Brito L., Novais P., Neves J., On the Logical Aspects of Argument-based Negotiation among Agents. Cooperative Information Agents IV (CIA2001), Klush, M., Zambonelli, F., (eds.), Lectures Notes in Computer Science Vol. 2182, Springer-Verlag (2001).

Faratin, P., Sierra, C., Jennings, N., Negotiation Decision Functions for Autonomous Agents. Proceedings of the International Journal of Robotics and Autonomous systems (1997), 25(3-4).

Jennings, N. R., Parsons, S., Noriega, P., Sierra, C., On Argumentation-Based Negotiation, Proceedings of the International Workshop on Multi-Agent Systems, USA (1998).

Kephart, J., Hanson, J., Greenwald, A., Dynamic Pricing by Software Agents, in Computer Networks (2000).

Norman, T., Sierra, C., Jennings, N., Rights and Commitment in Multi-Agent Agreements. Proceedings of the third International Conference on Multi-Agent Systems - ICMAS 1998, pp 222-229, France (1998).

Neves, J., Machado, J., Analide, C., Novais, P., Abelha, A., Extended Logic Programming Applied to the Specification of Multi-Agent Systems and Their Computing Environments. Proceedings of the 1997 IEEE International Conference on Intelligent Processing Systems, China (1997).

Novais, P., Brito, L., Analide, C., Neves, J., Argumentum to Argumenta: Using Logic for Negotiation in Electronic Commerce. Technical Report, Portugal (2002).

Novais, P., Brito, L., Neves, J., Developing Agents for Electronic Commerce - A Constructive Approach. Proceedings of the SCI 2001 - 5th World Multiconference on Systemics, Cybernetics and Informatics, USA (2001).

Novais, P., Brito, L., Neves, J., Experience-Based Mediator Agents as the Basis of an Electronic Commerce System. Proceedings of the Workshop 2000 - Agent-Based Simulation, Germany (2000).

Novais, P., Brito, L., Neves, J., Agreement in Virtual Marketplaces with CBR-Supported Negotiation. Proceedings of the Fifth International Conference and Exhibition on The Pratical Application of Intelligent Agents and Multi-Agents - PAAM 2000, UK (2000).

Prakken, H., Logical Tools for Modelling Legal Argument. Doctoral Dissertation, Free University, Amsterdam (1993).

Sartor, G., A Formal Model of Legal Argumentation. Ratio Juris 7, (1994). 